\title{
Microsociología del estigma: aportes de Erving Goffman a la conceptualización psicosociológica del estigma social
}

\author{
Microsociology of stigma: Erving Goffman's contributions to the psychosociological \\ conceptualization of social stigma
}

\author{
Marija Miric ${ }^{1}$ \\ José Luis Álvaro \\ Rafael González ${ }^{3}$ \\ Ana Raquel Rosas Torres ${ }^{4}$
}

\begin{abstract}
RESUMEN: Pese a su presencia temprana en las sociedades humanas, el estudio del estigma desde las ciencias sociales se inicia a mediados del siglo XX, constituyendo la definición de Erving Goffman del estigma como un atributo profundamente desacreditador dentro de una interacción social particular, una de las primeras aproximaciones al estudio sistemático y formal de este concepto. A diferencia de muchos autores contemporaneos, Goffman identifica oportunamente los rasgos propios del estigma social que permiten su clara delimitación con respecto a otros fenómenos vinculados a sus manifestaciones, siendo el reconocimiento de su singularidad conceptual el primer paso hacia el análisis y la compresión de sus complejas manifestaciones en diferentes contextos socio-culturales. La microsociología de Goffman y, de manera particular, su concepto de marcos, como esquemas generales que organizan la experiencia humana, ofrece un enfoque teórico fértil y, a la vez, poco explorado, para el análisis de este fenómeno y los mecanismos implicados tanto en su construcción social como en su impacto en los diferentes ámbitos de la interacción humana. Así, los marcos construidos, entre otros procesos, a partir de las narrativas y otras formas de comunicación popular, facilitan el reconocimiento y la codificación de objetos, situaciones, experiencias y secuencias de acciones desde el sistema de valores asumido por la comunidad, modificándose y ajustándose de manera continua a través del proceso de interacción social.
\end{abstract}

Palabras claves: estigma; discriminación; microsociología; marcos; Goffman.

ABSTRACT: Despite its early presence in human societies, stigma was not addressed by social sciences until mid-twentieth century, with Erving Goffman's definition of stigma as a profoundly discrediting attribute within a specific social interaction being one of the first attempts to produce a systematic and formal study of this phenomenon. Unlike many contemporary authors, Goffman accurately identifies features of social stigma that allow for its clear delimitation from other social phenomena related to its manifestations, understanding the acknowledgment of stigma's conceptual singularity as a first step required for analysis and comprehension of its complex manifestations in different socio-cultural contexts. Goffman's microsociology and, particularly, his concept of frames as schemata of interpretation that organize human experience, provide a fertile and, at the same time,

\footnotetext{
${ }^{1}$ Doctora en Psicología Social por la Universidad Complutense de Madrid. O\&M Medical School, Faculty of Health Sciences, Universidad Dominicana, O\&M - Santo Domingo, República Dominicana. E-mail: stella_mare@yahoo.com.

2 Doctor en Psicología Social por la Universidad Complutense de Madrid (UCM). Catedrático del Departamento de Psicología Social de la UCM - Madrid, España.

${ }^{3}$ Doctor en Psicología Social por la Universidad Complutense de Madrid (UCM). Profesor Titular del Departamento de Psicología Social de la UCM - Madrid, España.

${ }^{4}$ Doctora en Psicología Social por la Universidad de Kent (Canterbury). Profesora Titular de Psicología Social de la Universidad Federal de Paraiba. Brasil.
} 
relatively unexplored, theoretical ground for the analysis of social stigma, and the mechanisms involved both in its social construction and its impact in different areas of human interaction. From that perspective, frames constructed, among other social processes, through narratives and other forms of popular communication, facilitate the recognition and coding of objects, situations, experiences and sequences of actions based on the value system assumed by specific communities, being continually modified and adjusted through social interaction.

Keywords: stigma; discrimination; microsociology; frames; Goffman.

\section{Introducción}

El estigma como fenómeno social ha acompañado a las sociedades humanas desde sus orígenes, evidenciándose su presencia en múltiples fuentes históricas y literarias, en torno a características y condiciones que en determinados contextos sociales descalificaban a una persona o a un grupo de personas como miembros plenos y válidos de sus sociedades (Neuberg, Smith, \& Asher, 2000). No obstante, el reconocimiento de su relevancia y el interés en su estudio desde las ciencias sociales surge a mediados del siglo $\mathrm{XX}$, siendo la obra de Erving Goffman una de las primeras aproximaciones al estudio sistemático y formal de este fenómeno.

Si bien la obra de Erving Goffman abarcó una amplia gama de temas, su mayor impacto en el desarrollo de las ciencias sociales, en la segunda mitad del siglo XX, lo constituye el estudio de la interacción humana cara a cara en diferentes contextos sociales (Chriss, 1996). A partir de la publicación de su primera y posiblemente más conocida obra, The Presentation of Self in Everyday Life, Goffman (1959), basándose en la metáfora de teatro como herramienta analítica para la comprensión de la interacción social, pone el centro de atención en el manejo de impresiones en las relaciones cotidianas, en función de las percepciones que las personas tienen sobre las expectativas de otros actores involucrados en las mismas (Álvaro \& Garrido, 2003).

En 1963 su obra, Stigma: Notes on the Management of Spoiled Identity, marca el primer esfuerzo sistemático para estudiar el fenómeno del estigma desde las ciencias sociales. En este libro, basado en la observación participante y complementado con los relatos anecdóticos vinculados con los temas abordados, Goffman define el estigma como "un atributo profundamente desacreditador" (Goffman, 1963, p. 3) dentro de una interacción social particular.

Más allá de sus escritos enfocados de manera directa al análisis del estigma social, la microsociología del Goffman y, de manera particular, su concepto de marcos, como esquemas generales que organizan la experiencia humana (Goffman, 1974), ofrece un enfoque teórico fértil y, a la vez, poco explorado, para el análisis de este fenómeno y los mecanismos implicados tanto en su construcción social como en su impacto en los diferentes ámbitos de la interacción humana.

En este sentido, los marcos de Goffman pueden ser vistos como una aplicación microsociológica del concepto de marco de referencia originalmente concebido como un proceso psicológico por Gregory Bateson (1972), referido a la herramienta metacomunicacional que permite a todos los participantes en una interacción identificar su contexto relacional, facilitando los parámetros para la interpretación de la interacción simbólica y las acciones de otros integrantes de la misma. Así, los marcos construidos, entre otros procesos, a partir de las 
narrativas y otras formas de comunicación popular, facilitan el reconocimiento y la codificación de objetos, situaciones, experiencias y secuencias de acciones desde el sistema de valores asumido por la comunidad, modificándose de manera continua a través del proceso de interacción social (Goffman, 1963).

Con posterioridad a la obra inicial de Goffman y, especialmente, en las últimas décadas, el estudio del estigma ha ganado cada vez más importancia, no solamente dentro de las ciencias sociales, sino también en diferentes áreas de la salud, al observarse su importante impacto negativo para la planificación y la provisión de servicios, particularmente aquellos vinculados con las afecciones contagiosas y de transmisión sexual (Leary \& Schreindorfer, 1998). Esto ha contribuido a un notable cúmulo de datos y estudios empíricos sobre las principales características y manifestaciones del estigma social en diferentes ámbitos de la existencia humana, así como sobre las múltiples consecuencias, directas e indirectas, que puede tener este fenómeno para las personas y su vida en sociedad (Link \& Phelan, 2001; Miric, 2003a).

El escaso esfuerzo dedicado a la delimitación conceptual del estigma ha contribuido a un desbordamiento progresivo de las bases conceptuales propuestas por Goffman, incluyéndose en su definición una serie de fenómenos sociales vinculados empíricamente a sus manifestaciones, como son, por ejemplo, los de discriminación y exclusión social (Link \& Phelan, 2001), lo cual, más que enriquecer el concepto original ha llevado a definiciones cada vez más vagas e imprecisas del mismo (Miric, 2003b). Esta situación ha provocado el surgimiento reciente de un intento de delimitación del concepto de estigma retomando las bases teóricas planteadas en la obra de Goffman, como punto de partida para la construcción de un eje conceptual sólido para la sistematización e interpretación de diferentes estudios que tienen como objetivo principal su estudio. Estos esfuerzos, fundamentados en el estigma vinculado a diferentes condiciones de salud, identifican este fenómeno como uno de los determinantes sociales más relevantes de las desigualdades sociales en las sociedades contemporáneas (Weiss, Ramakrishna \& Somma, 2006; Hatzenbuehler, Phelan,\& Link, 2013; Phelan, Lucas, Ridgeway \& Taylor, 2014), analizando de manera prioritaria la importancia de su estudio en relación a las políticas públicas de salud (Link \& Hatzenbuehler, 2016).

Partiendo de este contexto, este artículo analiza las aportaciones teóricas de Goffman a la conceptualización del estigma, poniendo de manifiesto la relevancia de la microsociología para la construcción de enfoques teórico-empíricos que pretendan analizar y abordar este fenómeno desde las ciencias sociales. De manera particular, se toma en cuenta la teoría de marcos de Goffman y la definición del estigma social basada en este enfoque teórico, como un eje conceptual sólido y fértil para el estudio del estigma y sus múltiples manifestaciones en la sociedad contemporánea; particularmente, el impacto del estigma en la interacción social.

\section{Estigma social: propuesta de Goffman}

La obra de Goffman, cuya amplitud y originalidad hacen difícil su identificación con una sola de las grandes escuelas de pensamiento sociológico, refleja la influencia de varias corrientes, incluyendo el interaccionismo simbólico, la etnometodología, la sociología fenomenológica y la filosofía analítica (Álvaro \& Garrido, 2003). Asimismo, se han mencionado 
entre sus raíces intelectuales la antropología social inglesa, destacándose la influencia de Durkheim y Radcliffe-Brown (Collins, 1986), junto al posible impacto de las teorías freudianas en sus escritos (Bock, 1988).

Randall Collins (1986), uno de sus principales discípulos y el más activo de ellos, sistematiza el pensamiento de Goffman en tres grandes fases, en función del núcleo temático propio de cada una de ellas. Durante la primera fase, desarrollada en los años 50 y hasta mediados de la década de los 60, Goffman se dedicó a los rituales de la vida cotidiana, retomando la antropología social de Lloyd Warner y los estudios empíricos de la Escuela de Chicago. En esta época, además de su famosa obra, The Presentation of Self in Everyday Life, en la cual expone los fundamentos de su enfoque dramatúrgico, Goffman se dedicó al estudio de la desviación y el estigma social. A finales de los 60 y en los primeros años de la década de los 70, Goffman abordó el análisis del conflicto, propio de esa época. Así su obra Interacción Estratégica, publicada en 1969, está dedicada a las maniobras de negociaciones entre empresas, diplomacia internacional, etc. Finalmente, en la tercera fase, surge su interés por el pensamiento y el lenguaje, reflejado en sus últimas obras, desde Frame Analysis (1974) hasta Forms of Talk (1981), sin olvidar Gender Advertisements (1979) donde Goffman analiza numerosas fotografías en las que se muestran, con rotunda claridad, los patrones de diferencias de género observables en las representaciones publicitarias de los cuerpos de hombres y mujeres.

Si bien las aportaciones de Goffman más directamente dirigidas al estudio del estigma social pertenecen a la primera de estas fases, no cabe duda de que la teoría de los marcos, desarrollada en los estadios más avanzados de su obra, ofrece un eje teórico muy relevante para la comprensión de los mecanismos a través de los cuales el estigma construye e impacta las interacciones humanas, incluyendo su estrecho vínculo con el análisis de discurso como uno de los ejes centrales de este proceso.

Aun cuando antes de Goffman hubo autores que estudiaron la situación de individuos rechazados por sus grupos sociales, entre los cuales él mismo cita a Lemert, Lewin, Heider, Dembo, Barrer y Wright (Goffman, 1963), entre otros, su definición del estigma lo constituye como el pionero del estudio psicosociológico de este fenómeno. Con la publicación de su libro Stigma: Notes on the management of spoiled identity, Goffman se propone organizar el material previamente escrito sobre el tema dentro de un mismo esquema conceptual, y clarificar la relación entre estigma y desviación social.

Según Goffman, el estigma aparece durante las interacciones sociales, cuando la identidad social actual de un individuo - es decir, los atributos que posee - dejan de satisfacer las expectativas sociales (Goffman, 1963). A partir de este atributo, el individuo pasa a ser percibido como un individuo cuestionado, disminuido en su valor social. Distingue entre tres tipos de estigma, los cuales, no obstante, responden a mecanismos similares: deformaciones corporales; debilidades caracterológicas, como es el caso de trastornos mentales, encarcelamiento, adicción, alcoholismo, entre otras; y estigma tribal, referido a la raza, la nación y la religión.

Por otro lado, de manera irónica, tal y como señala Goffman, las personas estigmatizadas tienden a aceptar y asumir las mismas normas sociales que las estigmatizan y las descalifican para una participación social igualitaria. Al mismo tiempo, Goffman marca la diferencia entre el 
desacreditado y el desacreditable, dado que en esta segunda situación la persona es capaz de ocultar su estigma, por lo menos parcial y temporalmente. Esta posibilidad complica aun más el impacto del estigma en las interacciones sociales, estando la persona estigmatizada en situación de seleccionar a quienes y de qué manera desea revelar su estigma.

Es importante señalar que, a lo largo de su obra, Goffman identifica oportunamente algunas características del estigma social que pueden fundamentar su independencia conceptual de otros fenómenos vinculados a sus manifestaciones (Miric, 2003b). Se destacan, en este sentido:

a) La reacción emocional propia del estigma. Goffman (1963) señala y describe la reacción emocional que desencadena un atributo estigmatizante observable en una persona "normal", es decir, una persona que no porta este atributo, dentro de una interacción social, como una sensación de incomodidad más o menos intensa en la presencia de una persona estigmatizada. Stangor y Crandall (2000), entre otros autores posteriores a Goffman que abordan esta reacción, la describen como una activación fisiológica visceral, directa, y experimentada por el individuo como aversión o repugnancia frente al contacto inmediato con la persona estigmatizada. Esta reacción, que, aparentemente está ausente en el caso de las actitudes o los estereotipos negativos, alcanza su mayor intensidad frente a las deformidades físicas y diferentes tipos de enfermedades, y surge frente a todo atributo estigmatizante que sea observable o, de otra manera, conocido para el receptor.

b) Las racionalizaciones frente a la reacción emocional. Goffman llama la atención sobre la tendencia de las personas "normales" a acudir a diferentes ideologías que justifiquen el rechazo de las personas que portan un estigma social (Goffman, 1963). Estas racionalizaciones, designadas posteriormente por Stangor y Crandall (2000) como "ideologías de justificación", resultan, casi siempre, insostenibles frente a una argumentación racional, manteniéndose como un sistema de creencias no contrastadas y, en última instancia, no contrastables; cuando estas creencias se ven amenazadas, las personas que las comparten tienden a reaccionar con angustia, hostilidad y enojo.

c) Contagio. La naturaleza "contagiosa" del estigma, representa otra de las particularidades de este fenómeno identificada por Goffman. Mientras que las actitudes y los estereotipos negativos tienden a limitarse a las personas que poseen cierta característica 0 pertenecen a un grupo determinado, el estigma que padecen las personas portadoras de atributos estigmatizantes, sean éstos físicos o simbólicos, suele "transmitirse" a toda persona u objeto que entra en contacto con ellos.

d) Supresión del estigma. Este fenómeno, descrito por algunos autores como la inhibición del rechazo desencadenado normalmente por la reacción emocional negativa que provoca el estigma (Miric, 2003b), queda reconocido como uno de los aspectos que puede llegar a interferir en las interacciones entre una persona "normal" y una estigmatizada. Este tipo de interacciones da lugar a lo que Goffman (1963) denomina como "awkward moments", en los cuales todos los participantes se sienten incómodos (sin que necesariamente haya una intención de discriminar). 
Finalmente, cabe destacar la concepción de Goffman sobre la desviación social, la cual, según él, constituye un puente entre el estudio del estigma y el estudio del resto del mundo social (Goffman, 1963). Así, los mecanismos que subyacen al fenómeno del estigma no son esencialmente diferentes a los que subyacen el manejo de cualquier desviación social entre las personas "normales". Más bien, podría plantearse que Goffman llega a concebir el estigma como un punto determinado en el eje normalidad - desviación.

A pesar de la importante contribución de Goffman al estudio del estigma, sus análisis recibieron importantes críticas por parte de autores contemporáneos a la publicación de sus reflexiones. Se destaca en ese sentido la poca diferenciación entre los ejemplos y las tesis teóricas expuestas, lo cual le imprime un tono anecdótico a sus escritos. Entre otras críticas fue señalada su concentración en lo obvio, la omisión de materiales empíricos menos "populares" en los ejemplos citados, el manejo de la alusión en lugar del análisis y la falta general de sistematicidad en la exposición (Seeman, 1964).

En las demás recensiones que aparecieron inmediatamente después de la publicación de Stigma, resulta curioso observar de qué manera coexisten, de manera simultánea, la crítica y la admiración sociológica hacia su ya muy conocido autor, que - gracias a la iniciativa de Talcott Parsons - había recibido el prestigioso premio Mclver por The Presentation of Self. Uno de los primeros glosadores, Melvin DeFleur, define Stigma como "un pequeño y provocativo libro" que se puede leer tanto desde la "decepción" que puede producir la escasez de sus análisis teóricos, o "con el aprecio y admiración" de ese cautivador estilo, tan característico de Goffman, que todos los sociólogos habían disfrutado en The Presentation of Self (DeFleur, 1964). Un año después, Eugene Weinstein describe Stigma como "variaciones del gran tema" anunciado por su autor en The Presentation of Self, indicando que la distinción entre "desacreditado" y "desacreditable" ya había sido señalada por Goffman en un trabajo previo, Embarrassment and Social Organization, publicado en 1956 en The American Journal of Sociology. Stigma recupera dicha dualidad, aunque en este caso se concentra, únicamente, en las situaciones "permanentes" - y no reversibles, como en el caso de Embarrassment - en las que una persona puede sentirse desacreditable y desacreditada. $Y$, como no podía ser de otra manera, Weinstein (1965) manifiesta su "irritación" por el que considera un texto excesivamente "relleno" de anécdotas y ejemplos aparentemente desconectados entre sí, aunque reconoce que nadie es capaz de observar la interacción tan bien como lo hace Goffman. Algunos años después, Alvin Gouldner (1973) dedicará a Goffman buena parte del capítulo 10 de su libro La crisis de la sociología occidental. Aunque reconoce la brillantez y originalidad de todos y cada uno de los escritos de Goffman, y el merecido impacto que, desde sus primeras publicaciones, tuvo en la sociología estadounidense, Gouldner le reprocha con dureza la ambigüedad ideológica de la mayoría de sus escritos, su escasísimo interés por las consecuencias de la estratificación social o las posibles estrategias para defenderse de los abusos e injusticias del poder, excepto, claro está, cuando, de manera individual, alguien intenta preservar su dignidad e identidad personal, como se detalla a través de los innumerables ejemplos reunidos en Estigma.

Si bien muchas de estas críticas pueden considerarse oportunas y, efectivamente, las deficiencias referidas dificultan notablemente la identificación del eje conceptual que atraviesa esta obra de Goffman, es importante reiterar que se trata del primer antecedente formal del estudio del estigma social desde las ciencias sociales, el cual, además, estableció el camino para 
la conceptualización del estigma como un fenómeno social independiente, evitando la confusión conceptual propia de la mayor parte de los estudios posteriores (Miric, 2003a).

Por otro lado, si bien Goffman, no establece vínculos directos con conceptos derivados de otras perspectivas psicosociológicas (Psathas, 1996), sus propuestas relativas al estudio del estigma social se complementan con algunas de ellas. La integración de estos enfoques a la base conceptual propuesta por Goffman, permitirían ampliar la comprensión del fenómeno del estigma en el proceso de construcción de un eje conceptual para su estudio desde las ciencias sociales.

Así, al estudiar el impacto del estigma social en el contexto de las interacciones humanas, Goffman refleja claramente la influencia del Interaccionismo Simbólico (Álvaro \& Garrido, 2003), concibiendo el proceso de comunicación, situado en el contexto de interacción social entre dos o más individuos, como fundamento del proceso de la construcción social de este fenómeno. Asimismo, los planteamientos de Goffman sobre el impacto del estigma a través del proceso de comunicación quedan complementados y ampliados por los aportes de la Escuela de Palo Alto al estudio de la comunicación humana, indicando los posibles mecanismos específicos a través de los cuales un atributo estigmatizante logra impactar en las interacciones sociales en las cuales está presente (Watzlawick, Beavin \& Jackson, 1967).

Este vínculo entre el trabajo de Goffman, el Interaccionismo Simbólico y la Escuela de Palo Alto para la comprensión de la comunicación humana en el contexto de la interacción social, ha sido señalado por Rizo (2004) como la mayor contribución a la dimensión comunicológica de la interacción. Por otro lado, la concepción del estigma como un fenómeno construido socialmente a través del proceso de comunicación y en el contexto de la interacción social, vincula su comprensión desde las ciencias sociales a una de las premisas principales de la psicología social discursiva: el planteamiento de que los fenómenos que interesan a la investigación psicosocial están constituidos en y a través del discurso (Kroger \& Wood, 1998; Sampson, 1993).

Aplicando esta afirmación al estudio del estigma, el discurso construido al respecto, a través del proceso de comunicación, no es un mero reflejo de este concepto como una realidad previamente concebida, sino que, al contrario, constituye una re-construcción permanente del estigma como realidad social. González (2012) destaca la intencionalidad de agentes estigmatizadores y de un propósito estigmatizador, como elementos claves de este dinamismo del estigma social basado en el discurso construido desde posiciones de poder.

Una de las principales críticas que continúa recibiendo Goffman procede de científicos sociales que, cada vez con mayor actividad y presencia pública, desde la década de 1960, pertenecen a amplios colectivos que han soportado diferentes grados de estigmatización, como las mujeres, las minorías étnicas, las personas con enfermedad crónica o discapacidad y las organizaciones LGBT (lesbianas, gais, bisexuales y transexuales). Tal es el caso de autores como Susan Sontag (2003) y su denuncia de las metáforas estigmatizadoras de enfermedades como el cáncer o el SIDA, que muchas veces se interpretan como "castigos" a conductas de "riesgo" y moralmente "inapropiadas"; o Didier Eribon, historiador y militante LGBT, discípulo de Foucault, que desde hace varios años insiste en la necesidad de que los colectivos estigmatizados se comprometan activamente en defender -de manera enérgica- su derecho al respeto y a la 
dignidad. Según Eribon (2001), la lucha contra la injuria, las manifestaciones públicas de desprecio, o la indiferencia de buena parte de la opinión pública frente a las agresiones físicas que, con elevada frecuencia, son víctimas las personas estigmatizadas, requieren dos amplios tipos de medidas sociales: político-jurídicas (a través de la proclamación de leyes de igualdad que se hagan cumplir de manera efectiva por parte de los jueces), y socio-culturales (con la aplicación de amplias estrategias de naturaleza educativa para la promoción de la tolerancia, en las que también participen los medios de comunicación). Estos dos tipos de medidas deben desarrollarse de manera complementaria, a través de a) leyes de Derechos Civiles, y b) normas para la "visibilización" de las minorías en programas televisivos de ficción. En Estados Unidos se fueron aplicando ambas estrategias entre 1964 y 1974, y en la actualidad todas las series de televisión ya integran en su reparto a actores negros, hispanos, asiáticos, LGBT, etc., siendo cada vez más frecuente que el papel de jueces, fiscales o directores de servicios secretos esté interpretado por mujeres. Ahora bien, ambas medidas resultarán escasamente eficaces sin la colaboración activa y sincera del resto de los ciudadanos, lo que, como señalaron Allport (1954) y Moscovici (1976) no resulta fácil de conseguir. En el mejor de los casos, las personas estigmatizadas quedarán a salvo de las manifestaciones públicas más "hostiles" y directas, pero permanecerán, entre muchas personas, sentimientos reprimidos de miedo y/o desprecio, o comportamientos "sutiles" de estigmatización en las "nuevas" formas de racismo, sexismo, etc. (Pettigrew \& Meertens, 1995).

En este sentido, tanto la comprensión del estigma como fenómeno social construido en y a través del discurso, como el diseño de estrategias dirigidas a la reducción de su impacto negativo en los diferentes ámbitos de la interacción humana, han de basarse en un análisis del discurso vinculado a sus manifestaciones.

Finalmente, el análisis del estigma en la comunicación humana, desde la teoría de los marcos (Goffman, 1974), guarda una relación estrecha con el concepto de representación social (Mora, 2002; Moscovici, 1961, 1988). No obstante, si bien la teoría de las representaciones sociales podría dar cuenta teórica de las actitudes frente a las personas que muestran un atributo desacreditador o estigmatizante, la teoría de los marcos propuesta por Goffman desde un enfoque micro-sociológico (Alzate, 2001; Bock, 1988; Psathas, 1996), permite conceptualizar e interpretar el estigma social en el contexto de la interacción de grupos pequeños, de manera compatible con la definición de este fenómeno como un atributo profundamente desacreditador (Goffman, 1963).

\section{Desarrollos posteriores en el estudio del estigma social}

Los autores posteriores a Goffman, en su mayoría, han abordado el fenómeno del estigma desde un plano básicamente descriptivo, cada uno desde su área de conocimiento, provocando una acumulación de información empírica, pero sin contar con un eje conceptual sólido que sirva de base para la sistematización y comprensión del mismo (Miric, 2003b).

Paralelamente a esta acumulación de datos sobre el fenómeno del estigma, se ha observado la tendencia a incluir en su definición otros fenómenos socio-culturales que, según lo observado, tienden a aparecer relacionados con un atributo estigmatizante. A partir de ahí, las 
críticas más frecuentes a las posiciones de Goffman sobre el estigma han girado en torno a su concentración en el atributo estigmatizante, que algunos autores han considerado como secundario a otros factores de corte interaccional y socio-cultural. Así, entre otros, se ha planteado que la concepción teórica de Goffman, además de implicar una concepción individualista del ser humano, no toma en cuenta los mecanismos a través de los cuales la cultura es reproducida por cada individuo que forma parte de ella (Das, 2002). Esta crítica, que infiere una posición individualista de Goffman a partir de su análisis de la sociedad desde la perspectiva microsociológica no coincide con la preponderancia otorgada por este autor a la sociedad frente al individuo (Álvaro \& Garrido, 2003; Collins, 1986).

Por otro lado, hay que tener en cuenta que la atención que pone Goffman en el atributo estigmatizante radica en el hecho de que, para él, el estigma es este atributo, y no el proceso de interacción social del cual este atributo forma parte, como lo asume una gran parte de autores posteriores a él. Goffman no solamente afirma la importancia de los factores socio-culturales en el estudio de estigma, sino que insiste en que un atributo potencialmente estigmatizante está siempre enraizado en los significados compartidos culturalmente, siendo imposible desvincularlo de un periodo histórico particular y un contexto cultural determinado (Alonzo \& Reynolds, 1995). Por otro lado, a lo largo de su obra Goffman señala repetidamente la importancia de factores culturales y de interacción vinculados al fenómeno de estigma, sosteniendo que éste representa un lenguaje de relaciones, donde un atributo determinado, que puede estigmatizar a un grupo de individuos, puede servir como confirmatorio de la 'normalidad' de otro, y no es, por tanto, ni positivo ni negativo en sí mismo (Goffman, 1963). Este hecho queda debidamente reconocido en el importante artículo de Parker y Aggleton (2003) dedicado a la conceptualización del estigma, cuando señalan que la concepción del estigma como atributo fue sacado del contexto de la obra de Goffman en muchos trabajos posteriores sobre este tema.

Resumiendo lo anterior, puede plantearse que la diferencia esencial de las posiciones de Goffman con respecto a las tendencias posteriores en el análisis del estigma, no radica en su descuido de las variables socio-culturales que acompañan a este fenómeno, sino en la no inclusión de estas variables como integrantes del concepto mismo de estigma. Sin duda alguna, los intentos posteriores a Goffman de contemplar como parte del estigma los fenómenos empíricamente asociados a los atributos estigmatizantes en determinados contextos socioculturales han dificultado más aun la aproximación a una definición conceptual precisa de estigma en las últimas décadas. Las bases conceptuales formuladas por Goffman han perdido progresivamente sus límites, desbordándose así el concepto de estigma en una confusión general de fenómenos y conceptos (Miric, 2003b).

Esta confusión general quedó reflejada en las definiciones de estigma cada vez más descriptivas, generales e imprecisas, asumidas por una gran parte de estudios empíricos sobre el estigma realizados en las últimas décadas. Algunos de ellos han estado basados en las definiciones de estigma como "un concepto amplio y multidimensional cuya esencia está centrada en la desviación social" (Alonzo \& Reynolds, 1995, p.307) o como algo que está presente cuando "nos referimos a todo grupo de personas evaluadas negativamente, ya sea por haber violado reglas sociales, o por ser como son y portar rasgos que no son positivamente evaluados por el resto de la sociedad" (Birenbaum \& Sagarin, 1975 p. 205). Más aún, no 
escasean investigaciones dedicadas al estudio del estigma que lo reducen a un sinónimo de discriminación y exclusión social. Si se concibe un grupo estigmatizado como "una categoría de personas a quienes la sociedad más amplia se refiere peyorativamente y que son devaluadas, excluidas 0 , de alguna otra manera, inhibidas en cuanto a sus posibilidades de vida y acceso a las ventajas de una interacción social no restringida y libre" (Alonzo \& Reynolds, 1995, p.305), ¿cómo establecer la distinción entre un grupo estigmatizado y un grupo socialmente discriminado? Por otro lado, de no poder establecerse esta distinción, ¿cómo justificar el empleo del término "estigma" frente a otro como el de "discriminación social"?

En respuesta a esta situación de ambigüedad conceptual en torno al fenómeno del estigma, recientemente han surgido publicaciones encaminadas hacia una nueva concepción del mismo, que permita conciliar, unificar y sintetizar las observaciones e inferencias realizadas en el transcurso de las últimas décadas, con frecuencia muy discrepantes entre sí. Estas publicaciones están, en gran parte, motivadas y referidas al impacto del estigma en planificación y provisión de servicios en salud pública, puesto en evidencia de manera especial en atención a enfermedades contagiosas y de transmisión sexual, como es el caso del VIH y el SIDA.

Entre estas publicaciones se destaca el trabajo de Link y Phelan (Link \& Phelan, 2001, 2006; Phelan et al., 2014; Link \& Hatzenbuehler, 2016), quienes amplian la idea original de Goffman, proponiendo concebir el estigma, no como un atributo, sino como un proceso social. Según estos autores, quienes enfocan de manera prioritaria la intersección entre las manifestaciones estigmatizantes y las políticas públicas, el proceso de estigmatización comienza cuando los grupos dominantes de una sociedad reconocen ciertas diferencias humanas - sean éstas verdaderas o no; continua, si se considera que las diferencias observadas implican información desfavorable sobre las personas designadas; en la medida en que ocurre esto, se consigue su rotulación social a partir de estas diferencias. Las personas rotuladas son clasificadas en una categoría aparte, separando así el "nosotros" de "ellos" (Link \& Phelan, 2001). La culminación del proceso de estigmatización ocurre cuando las diferencias designadas desencadenan distintas formas de desaprobación, rechazo, exclusión y discriminación. Esta visión del estigma como proceso permite integrar en un todo coherente los fenómenos que aparecen vinculados a los atributos estigmatizantes, manteniendo a la vez intactos los límites entre ellos. Así, para Link y Phelan (2001) los fenómenos como la desaprobación, rechazo, exclusión y discriminación forman parte del proceso de estigma, pero no se confunden con el estigma mismo, siendo éste un concepto más amplio; representan únicamente su fase de culminación.

Resumiendo lo anterior, el término "estigma" queda referido a todo el conjunto de fenómenos que intervienen en el impacto social de un atributo estigmatizante, en lugar de limitarlo al atributo en sí, como hacía Goffman. Sin embargo, estudiando más detenidamente los cambios introducidos de esta manera, se observa que son más bien de carácter terminológico. En este sentido, Link, Phelan y otros autores posteriores a Goffman no añaden ningún elemento nuevo para la comprensión del proceso que designan como "estigma", ni acerca de los mecanismos por los cuales se desencadena, limitándose a describir las diferentes fases del mismo. 
Tomando esto en cuenta, definir el estigma como un proceso social, ampliando el concepto original para incluir los fenómenos sociales vinculados, complejiza su análisis sin aportar nuevas perspectivas teóricas para su comprensión. El estigma, sin duda alguna, forma parte y logra su impacto a través de un complejo proceso social, previamente descrito por Goffman y compatible con los planteamientos de Link \& Phelan (2001, 2006, 2014), íntimamente vinculado con la comunicación social. No obstante, resulta, si no equívoco, cuando menos poco productivo, asignarle el nombre de estigma a este proceso en su totalidad; más bien, sin descuidar el estudio de otros eslabones de este proceso, el estigma ha de ser comprendido como un fenómeno social independiente antes de poder ser abordado como integrante de un proceso social.

\section{Conclusiones}

Partiendo de lo anteriormente descrito, es evidente la relevancia de la microsociología de Goffman para la construcción de enfoques teórico-empíricos que pretendan analizar y abordar el fenómeno del estigma desde las ciencias sociales, asumiéndolo como un atributo profundamente desacreditador cuya presencia impacta la comunicación y la interacción humana, quedando reflejado y, a la vez, reforzado, por los discursos propios de cada contexto cultural. Así, el concepto de marco de Goffman facilita la comprensión del impacto psicológico del estigma desde la teoría de comunicación humana y los postulados de la Escuela de Palo Alto, permitiendo, a la vez, abordar estos mecanismos desde un enfoque psicosociológico compatible con los fundamentos del interaccionismo simbólico, en el contexto de la comunicación humana y la interacción en grupos pequeños.

Desde esta perspectiva, podría plantearse que un atributo pasa a ser estigmatizante - y es interpretado como tal - en el contexto de una interacción social particular, a partir de los marcos de referencia que orientan dicha interacción. De manera compatible con el Interaccionismo Simbólico, estos marcos quedan construidos y delimitados, entre otros procesos, a partir de las narrativas y otras formas de comunicación popular, facilitando el reconocimiento y la codificación de objetos, situaciones, experiencias y secuencias de acciones desde el sistema de valores asumido por la comunidad, y modificándose de manera continua a través del proceso de interacción social. De ahí que, por ejemplo, las narrativas populares que presentan a personas que viven con el VIH como individuos resentidos, malintencionados y peligrosos, que pretenden transmitir este virus a otras personas de manera intencional, podrían contribuir a la atribución simbólica de la "mala fe" a las personas que viven con esta condición de salud y su evitación consecuente en las interacciones cotidianas.

Si bien es cierto que el frecuente carácter anecdótico de sus observaciones y el estilo propio de sus escritos sobre el tema del estigma dificultan la identificación del eje conceptual que las subyace, Goffman, sin duda alguna, proporciona las premisas y abre el camino hacia la comprensión de este fenómeno y su fundamentación conceptual desde las ciencias sociales. Más aún, a diferencia de muchos autores más recientes, Goffman identifica oportunamente los rasgos propios del estigma social que permiten su clara delimitación con respecto a otros fenómenos vinculados a sus manifestaciones. El reconocimiento de la autonomía conceptual del estigma es el primer paso hacia el análisis y compresión de sus complejas manifestaciones 
en diferentes contextos socio-culturales y de su relación con otras dimensiones de interacción humana.

\section{Referencias}

Allport, G. (1954). La naturaleza del prejuicio. Buenos Aires. Paidós: 1971.

Alonzo, A., \& Reynolds, N. (1995). Stigma, HIV and AIDS: an Exploration and Elaboration of a Stigma Trajectory. Social Science and Medicine, 41(3), 3-20. DOI: https://doi.org/10.1016/0277-9536(94)00384-6.

Alzate, F. H. (2001). Goffman y el agenciamiento microsociológico. Poiésis, 1(2), 1-2. DOI: https://doi.org/10.21501/16920945.1098.

Álvaro, J. L., \& Garrido, A. (2003). Psicología social: perspectivas psicológicas y sociológicas. Madrid: McGraw-Hill.

Bateson, G. (1972). Steps to an Ecology of Mind: Collected Essays in Anthropology, Psychiatry, Evolution, and Epistemology. Chicago: University of Chicago Press.

Birenbaum, A. (1975). The disabled as involuntary deviants. In E. Sagarin (Ed.). Deviants and deviance: An introduction to the study of disvalued people and behavior (pp. 201-214). Westport, CT: Praeger.

Bock, P. K. (1988). The importance of Erving Goffman to psychological anthropology. Ethos, 16(1), 3-20. DOI: 10.1525/eth.1988.16.1.02a00010.

Chriss, J. J. (1996). Toward an Interparadigmatic Dialogue on Goffman. Sociological Perspectives, 39(3), 333-339.

Collins, R. (1986). The Passing of Intellectual Generations: Reflections on the Death of Erving Goffman. Sociological Theory, 4(1), 106-113.

Das, V. (2002). Stigma, Contagion, Defect: Issues in the Anthropology of Public Health. Presented at the International Conference on Stigma and Global Health: Developing a Research Agenda. Consultado en http://www.stigmaconference.nih.gov/FinalDasPaper.htm.

DeFleur, M. L. (1964). "Stigma: Notes on the Management of the Spoiled Identity. By Erving Goffman". Social Forces, 43(10), 127-128. Consultado en https://academic.oup.com/sf/articleabstract/43/1/127/2227971?redirectedFrom=fulltext

Eribon, D. (2001). Reflexiones sobre la cuestión gay. Barcelona: Anagrama.

Goffman. E. (1956) Embarrassment and Social Organization. American Journal of Sociology, 62(3), 264-271. DOI: https://doi.org/10.1086/222003.

Goffman, E. (1959). The Presentation of Self in Everyday Life (1a ed). New York: Anchor.

Goffman, E. (1963). Stigma: Notes on the Management of Spoiled Identity. Reissue ed: New York: Touchstone.

Goffman, E. (1969). Strategic Interaction. Philadelphia: University of Pennsylvania Press.

Goffman, E. (1974). Frame Analysis: An Essay on the Organization of Experience (1a ed.). Cambridge, Mass: Harvard University Press.

Goffman, E. (1979). Gender advertisements. Nueva York: Harper \& Row.

Goffman, E. (1981). Forms of Talk. Philadelphia: University of Pennsylvania Press.

González, R. (2012). Más allá de la psicologización: estigmatizaciones naturalizadoras individuales y colectivas. Teoría y Crítica de La Psicología, 2, 49-62.

Gouldner, A. (1973) La crisis de la sociología occidental. Buenos Aires: Amorrortu. 
Hatzenbuehler, M. L., Phelan, J. C., \& Link, B. G. (2013). Stigma as a fundamental cause of population health inequalities. American Journal of Public Health, 103(5), 813-821. DOI: 10.2105/AJPH.2012.301069.

Kroger, R. O., \& Wood, L. A. (1998). The turn to discourse in social psychology. Canadian Psychology/Psychologie Canadienne, 39(4), 266-279.

Leary, M. R., \& Schreindorfer, L. S. (1998). The stigmatization of HIV and AIDS: Rubbing salt in the wound. In Derlega, V. \& Barbee, A. (Eds.). HIV \& Social Interaction (pp. 12-29). California: SAGE Publications.

Link, B. G., \& Phelan, J. C. (2001). Conceptualizing Stigma. Annual Review of Sociology, 27, 363-385.

Link, B. G., \& Phelan, J. C. (2006). Stigma and its public health implications. The Lancet, 367(9509), 528-529. DOI: http://dx.doi.org/10.1016/S0140-6736(06)68184-1.

Link, B. G., \& Phelan, J. (2014). Stigma power. Social Science \& Medicine, 103, 24-32. DOI: https://doi.org/10.1016/j.socscimed.2013.07.035.

Link, B., \& Hatzenbuehler, M. L. (2016). Stigma as an unrecognized determinant of population health: research and policy implications. Journal of Health Politics, Policy and Law, 41(4), 653-673. DOI: 10.1215/036168783620869.

Miric, M. (2003a). Las huellas del tabú: Aproximaciones al concepto de estigma. Paradigmas, 1(1), 50-62.

Miric, M. (2003b). Estigma y discriminación: Vinculación y Demarcación. Paradigmas, 1(2), 83-97.

Mora, M. (2002). La teoría de las representaciones sociales de Serge Moscovici. Athenea Digital, 2(7), 1-25.

Moscovici, S. (1961). La psychanalyse, son image et son public. Paris: Presses Universitaires de France.

Moscovici, S. (1976). Psicología de las minorías activas. Madrid: Morata.

Moscovici, S. (1988). Notes towards a description of social representations. European Journal of Social Psychology, 18(3), 211-250. DOI: 10.1002/ejsp.2420180303.

Neuberg, S. L., Smith, D. M., \& Asher, T. (2000). Why people stigmatize: Toward a biocultural framework. In Heatherton, T. F. et al. (Orgs.). The social psychology of stigma (pp. 31-61). New York: The Guilford Press.

Parker, R., \& Aggleton, P. (2003). HIV and AIDS-related stigma and discrimination: a conceptual framework and implications for action. Social Science and Medicine, 57, 13-24. DOI: https://doi.org/10.1016/S02779536(02)00304-0.

Pettigrew, T. F., \& Meertens, R. W. (1995). Subtle and blatant prejudice in Western Europe. European Journal of Social Psychology, 25(1), 57-75. DOI: 10.1002/ejsp.2420250106.

Phelan, J. C., Lucas, J. W., Ridgeway, C. L., \& Taylor, C. J. (2014). Stigma, status, and population health. Social Science \& Medicine, 103, 15-23. DOI: https://doi.org/10.1016/j.socscimed.2013.10.004.

Psathas, G. (1996). Theoretical Perspectives on Goffman: Critique and Commentary. Sociological Perspectives, 39(3), 383-391.

Rizo, M. (2004). El Camino Hacia la "Nueva Comunicación": breve Apunte Sobre las Aportaciones de la Escuela de Palo Alto. Razón Y Palabra, 40. Consultado en https://dialnet.unirioja.es/servlet/articulo?codigo=962386.

Sampson, E. E. (1993). Identity politics: Challenges to psychology's understanding. American Psychologist, 48(12), 1219-1230. DOI: http://dx.doi.org/10.1037/0003-066X.48.12.1219.

Seeman, M. (1964). Review: Stigma: Notes on the Management of Spoiled Identity". American Sociological Review, 29(5), 770-771.

Sontag, S. (2003). La enfermedad y sus metáforas. El sida y sus metáforas. Madrid: Punto de Lectura.

Stangor, C., \& Crandall, C. (2000). Threat and the Social Construction of Stigma. In Haetherton et al. (Orgs.). The Social Psychology of Stigma (pp. 62-88). New York:The Guilford Press.

Watzlawick, P., Beavin, J. H., \& Jackson, D. (1967). Teoría de la comunicación humana: interacciones, patologías y paradojas. Barcelona: Herder. 
Weinstein, E. (1965). Stigma: Notes on the Management of the Spoiled Identity. By Erving Goffman. The American Journal of Sociology, 7(5), 636.

Weiss, M. G., Ramakrishna, J., \& Somma, D. (2006). Health-related stigma: rethinking concepts and interventions. Psychology, Health \& Medicine, 11(3), 277-287. DOI: http://dx.doi.org/10.1080/13548500600595053.

Apresentação: 01/12/2017 Aprovação: 28/12/2017 